\title{
New Modalities of Treatment for Coronary Calcific Lesions
}

\author{
TNC Padmanabhan ${ }^{1}$ Mohammed Sadiq Azam \\ ${ }^{1}$ Department of Cardiology, KIMS, Secunderabad, Telangana, India
}

\begin{abstract}
Address for correspondence TNC Padmanabhan, MD DM, Department of Cardiology, KIMS, Secunderabad, Telangana, 500003, India (e-mail: tncpad@gmail.com).
\end{abstract}
Abstract
Keywords
- calcific coronary lesions
- imaging
- treatment

Since the publication of the previous article on the same topic in the journal, as a result of the better understanding in intravascular imaging (intravascular ultrasound [IVUS] and optical coherence tomography [OCT]) and introduction of intravascular lithotripsy (IVL) into clinical practice, the outcomes in patients with calcified coronary lesions have become more predictable and safe with lesser complication rates.

\section{Introduction}

Interventional treatment of heavily calcified lesions remains the Achilles heel of an interventional cardiology even today. Attempts at overcoming this challenge are fraught with a higher risk of immediate complications such as inability of the stent to cross, delamination of polymer, altered or improper drug elution, inadequate stent expansion and balloon rupture with occasional coronary perforations, as well as late failures due to stent underexpansion and malapposition, and consequent poor outcomes. ${ }^{1}$ As many as 30 to $35 \%$ cases presenting with acute coronary syndromes have calcific coronary stenoses, making their management even more challenging. ${ }^{2}$

Since the original description of coronary "ossification" by Edward Jenner and Caleb Hillier, we have come a long way in understanding the pathogenesis and characterizing coronary calcium. Proper characterization of coronary calcium by multimodal imaging with subsequent optimal preparation of the lesion "bed" are essential prerequisites for success while treating a calcific lesion. The interventionalist's armamentarium is today well-equipped with a wide array of devices to tackle coronary calcium. This article will focus on the newest modalities available at our disposal as of now to tackle these lesions.

\section{Pathophysiology of Calcified Plaque}

The mechanism of coronary artery calcification differs considerably compared with peripheral arterial calcification. In peripheral arteries, calcification in media is usually mediated by osteoblast-like cells acting on a stimulus of changes in systemic calcium and phosphate homeostasis. Atherosclerotic intimal coronary calcification, on the contrary, is due to dysmorphic calcium deposition by chondrocyte-like cells for which the stimulus is inflammatory cytokines from tissue macrophages and foam cells. The key pathophysiological factor is likely to be inflammation that begets calcification which, in turn, begets more inflammation leading to a vicious cycle.

The initial lesions are foci of microcalcifications (which are only detected on histopathology) over areas ranging from 0.5 to $15 \mu \mathrm{m}$ in size. These areas of microcalcifications can fuse into larger masses to become speckles and calcified sheets, which are detected in vivo by CT or intravascular imaging. Large protruding subintimal masses may lead to plaque destabilization and thrombus formation and has been detected in $5 \%$ cases of unstable coronary syndrome and ST-elevation myocardial infarction (STEMI) (the calcific nodule). Statin therapy promotes replacement of the fibronecrotic core within a plaque by fibrocalcific tissue. ${ }^{3-7}$
DOI https://doi.org/ 10.1055/s-0040-1722558.
C 2020 . Women in Cardiology and Related Sciences.

This is an open access article published by Thieme under the terms of the Creative Commons Attribution-NonDerivative-NonCommercial-License, permitting copying and reproduction so long as the original work is given appropriate credit. Contents may not be used for commercial purposes, or adapted, remixed, transformed or built upon. (https://creativecommons.org/licenses/by-nc-nd/4.0/).

Thieme Medical and Scientific Publishers Pvt. Ltd. A-12, 2nd Floor, Sector 2, Noida-201301 UP, India 


\section{Coronary Calcium and Events}

Coronary artery calcium score by $\mathrm{CT}$ was an independent predictor of coronary events irrespective of symptom status in the multi-ethnic study of atherosclerosis (MESA) study of 10-year follow-up. The SYNTAX score, a method to quantify the complexity of coronary lesions, also places high importance to the presence of heavy calcification (which is given 2 pts per lesion). Severity of calcification is greater with advanced age, hypertension, dyslipidemia, diabetes, and chronic kidney disease., ${ }^{8,9}$

\section{Assessment of Coronary Calcium}

\section{CT Coronary Artery Calcium (CAC) Score}

Formal calcium scoring was introduced in 1990 and has proven to be simple, elegant, reliable reproducible tool in predicting adverse outcomes. Agatston score was originally proposed for electron beam CT but was later adopted to multidetector CT using $120 \mathrm{KVp}$ with variable $\mathrm{mA}$ and $2.5 \mathrm{~mm}$ slice thickness. It is totaled score of all calcified lesions and all total calcified areas and maximum calcium density (>130 HU). Other scores are volume score and mass score. They are upwardly weighted with calcium density and fail to capture regional distribution of calcium with no account of number or size of calcified lesions. A score which accounts for calcium density and regional distribution, focal versus diffuse, number of lesions, lesion size, and microcalcification will refine the predictability. Some CAC features may be protective against the risk of atherosclerosis. In the MESA study, there was an inverse association between CAC density and cardiovascular disease (CVD) outcomes. ${ }^{10,11}$

The ability to detect vulnerable plaque by $\mathrm{CT}$ is limited. In the ICONIC nested case control arm of the CONFIRM registry (189 pts in each group), $1 \mathrm{k}$ plaque $(1000 \mathrm{Hu})$ is associated with lower risk of future acute coronary syndrome (ACS) during a follow-up period of around 3.9 years. ${ }^{12}$

\section{Fluoroscopy-Coronary Angiography}

Coronary calcification could be present in 8 to $32 \%$ patients undergoing coronary intervention. Coronary angiography often underestimates calcium severity and depth of calcium within the plaque. Radiopacity observed only during the cardiac cycle before injection of contrast medium is considered moderate by angiography. Radiopacity observed without cardiac motion, visible on both sides of the arterial lumen, as a double track (tram track calcification) is considered severe angiographically. Sensitivity of fluoroscopy for calcium detection is $40.2 \%$, intravascular ultrasound (IVUS) $82.7 \%$, and optical coherence tomography (OCT) $76.8 \%$ in one study. ${ }^{12}$ Culprit lesion calcification was severe in 402 patients (5.9\%), moderate in 1,788 (26.1\%), and none/mild in 4,665 (68.1\%) patients of ACS. ${ }^{2}$

Noninvasive assessment of coronary calcification like fluoroscopy and CT scan are suboptimal in guiding treatment modalities. IVUS can give idea of the arc and length of calcium but does not measure thickness of calcium correctly (due to acoustic shadowing). OCT gives better information of calcium distribution. Fugino has proposed a scoring system for OCT, which can aid in device selection for lesion preparation. Two points for maximum angle $>180^{\circ}, 1$ point for maximum thickness $>0.5 \mathrm{~mm}$, and 1 point for length $>5 \mathrm{~mm}$. A lesion score of 4 was associated with poor stent expansion. ${ }^{13}$

The advantages and limitations of various modalities of delineating coronary calcium are depicted in -Table $\mathbf{1}$.

\section{Statins and CAC}

Several randomized trials have shown that statins, despite being beneficial in preventing CVD events, were associated with progression of CAC. Volume of CAC is lessened, but density has increased with higher Agatston score, as higher weightage was given to density. There was a reduction in plaque volume and increase in calcium density by IVUS. Patients with gain of function mutation in PCSK-9 gene have extensive CAC and high prevalence of coronary artery disease (CAD). PCSK 9 inhibitors have no effect on CAC. Puri et al assessed plaque volume and calcification by IVUS in patients receiving high-dose statin, low-dose statin and no-statin therapy, and found that statins may stabilize plaques by calcifying necrotic core with reduction in coronary events. ${ }^{14}$ Consequently, the CAC score calculated by $\mathrm{CT}$ might be an imprecise modality in prognosticating patients on statins.

Table 1 Imaging techniques for coronary calcium detection: pros and cons

\begin{tabular}{|l|l|l|l|}
\hline Diagnostic accuracy & Angiography & IVUS & OCT \\
\hline Severe calcification & +++ & +++ & +++ \\
\hline Mild/moderate calcification & + & ++ & +++ \\
\hline Deep calcium & + & ++ & ++ \\
\hline Calcium arch & - & - & +++ \\
\hline Calcium thickness & - & + & +++ \\
\hline Longitudinal calcium length & - & +++ & + \\
\hline Nonhomogenous plaque/necrotic core & & + \\
\hline+++ Optimal ++: Moderate +: Modest & & + \\
\hline
\end{tabular}

Abbreviations: IVUS, intravascular ultrasound; OCT, optical coherence tomography. 


\section{Strategies to Tackle Calcified Coronary Lesions}

"Failing to prepare is preparing to fail"-John Wooden

The choice of access site depends on the experience and comfort of the operator. Many centers (including ours) have today shifted to an exclusive radial first approach, while some still swear by femoral access. Success in tackling a calcified lesion depends hugely upon planning the approach and strategies. The choice of guide, wires, balloons, and other specialized hardware like guide extensions, buddy wire, anchor balloon contributes a great deal toward success or failure. There are many specialized devices available today to tackle calcified lesions, which can be broadly classified as ablation techniques (rotational/orbital atherectomy), balloon-based techniques (cutting/scoring balloon, super high pressure balloon), excimer laser and lithotripsy balloon. Of these, those which are available at our disposal for routine clinical practice as of today are discussed here.

\section{High and Very High Pressure Noncompliant Balloons}

"When in doubt, use brute force" -Ken Thompson

Noncompliant (NC) balloons are characterized by a resilience to tolerate high inflation pressures with just a small increase in their diameter. This property allows the use of higher pressures in a focal segment of a coronary without risk of dog-boning and subsequent complications like dissection and perforation. These are of maximum benefit in calcific lesions, where the calcium arc is $<90^{\circ}$ and hence form the first choice in such cases.

The OPN NC balloon (SIS medical) is a unique super high pressure balloon that is 0.014 " wire, $5 \mathrm{~F}$ guide compatible, twin layer taper tip design with a rated burst pressure of $35 \mathrm{~atm}$, linear compliance at $>40 \mathrm{~atm}$, and available in sizes of 1.5 to $4.5 \mathrm{~mm}$ ( $0.5 \mathrm{~mm}$ increments) and three lengths (10,15 and $20 \mathrm{~mm}$ ).

In a multicenter, retrospective observational study of 326 consecutive undilatable lesions (with NC balloons) in patients involving two groups (group $1<40$ atm, group $2>40$ atm inflation pressure), Secco et al found that $>40$ atm pressure was required in $46.9 \%$ pts. OPN balloon alone was adequate in 288 pts (90.5\%). Rotablator was used in $17.3 \%$ in group 1 pts versus $1.5 \%$ in group 2 . Coronary perforation occurred in 3 pts $(0.9 \%)$ all in very high pressure ( $>40 \mathrm{~atm}$ ) group prior to stenting without rupture of balloon. At mean follow-up of 14 months, net event free survival was $91.5 \%$, mortality of $1 \%$, and target lesion revascularization (TVR) of $6.4 \%{ }^{15}$

\section{Rotational Atherectomy}

Rotational atherectomy (RA) system (Boston Scientific, Marlborough, Massachusetts) has a high-speed, rotating, elliptical, diamond-coated burr that acts as an abrasive on calcific plaque. Available sizes of the metallic burr are from 1.25 to 2.5 $\mathrm{mm}$ in increments of $0.25 \mathrm{~mm}$. The burr is mounted over an advancer (RotaLink) driveshaft and connected to an external motor that converts compressed gas into rotational energy.
The Rota burr requires a dedicated 0.009-inch wire (Rotawire), which is flexible with minimal unfavorable wire bias. RA works on the principle of differential cutting-it preferentially ablates the fibrocalcific plaque tissue while sparing the compliant elastic tissue. The tissue is pulverized into 5 to $10 \mathrm{um}$ (about the size of an RBC) debris, which are released into distal microcirculation and can lead to slow flow phenomenon in as many as $20 \%$ cases in one series.

The recommended burr/artery ratio is $0.5: 0.6$, and a safe range of burr revolution speed is between 135,000 and $180,000 \mathrm{rpm}$. The major factors influencing the outcome after RA are the eccentricity of calcium, luminal area, burr size, and degree of guide wire bias. Complications of RA include burr entrapment, coronary dissection, perforation and transient atrioventricular block (especially with right coronary RA). The risks of atrioventricular block can be mitigated with the insertion of a temporary pacemaker or pharmacologically with aminophylline or atropine administration. With experience, the incidence of complications can be minimized. Avoiding lesions in tortuosity, allowing for adequate time between runs, and preventing burr deceleration $>5000$ rpm can reduce complications. ${ }^{16-21}$

\section{Cutting and Scoring Balloons}

The first cutting balloon (Flextome Cutting Balloon) was introduced in 1991 by Boston Scientific and was available in monorail or over-the-wire catheter. It consists of a NC balloon with a set of 3 micro blade longitudinally oriented on the surface, which produces incisions within the atherosclerotic plaque during balloon inflation. The microblades work by causing radial incisions on the media, which is presumed to reduce elastic recoil and neointimal proliferation. The microblades prevent balloon slipping, which is of advantage in cases of in-stent restenosis due to intimal hyperplasia. A cutting balloon has the dual advantages of a more controlled lesion pre dilatation at lower inflation pressure. The GLOBAL study failed to show superiority of cutting balloons over regular NC balloons in type A/B lesions and hence their usage has been limited to cases with severe calcification and in-stent restenosis. The new iteration with Wolverine technology is supposed to reduce the profile of cutting balloon.

Difficulty in crossing and tracking severely calcific lesions are considered frequent limitations of cutting balloons. The trackability of a cutting balloon maybe improved by a new generation of low-profile semicompliant balloons with a scoring element on the surface (AngioSculpt, Biotronik; Scoreflex, OrbusNeich; NSE Alpha, B Braun). Scoring balloons allow focal concentration of the force during inflation and decreases the chance of balloon slippage. The radial force during balloon inflation is mainly exerted on the scoring element, which is transmitted in turn to the vessel wall, leading to incisions in the atherosclerotic plaque. Prolonged inflation of a scoring balloon produces a distortion force capable of expanding a resistant calcified lesion, the so-called "creep phenomenon," which helps achieve adequate dilatation of severely calcified lesions. ${ }^{22}$ The nitinol element ensures balloon anchoring with a lower risk of "melon-seeding," 
dissection and perforation. Scoring balloons are more flexible compared with cutting balloons, have a better profile, and can achieve full expansion with a low-inflation pressure, which reduces the trauma to the vessel wall and minimizes the risk of coronary dissection..$^{22-31}$ - Fig. 1 shows the results of AngioSculpt in a calcific left anterior descending (LAD) artery lesion.

\section{Orbital Atherectomy (OA)}

It is a relatively newer treatment for management of coronary lesions with severe calcification. Similar to RA, it consists of a diamond coated $1.25 \mathrm{~mm}$ crown; however, contrary to RA burr, the OA crown is eccentrically mounted and is coated with diamond chips on both front and back, which permits bidirectional ablation with burr advancement and withdrawal. On account of this, entrapment of an OA burr is much less likely. The crown is advanced over a 0.012-inch wire (Viper wire), which has better maneuverability than the Rotawire. ${ }^{19,32,33}$

Centrifugal force generated during rotation pushes and compresses the crown against the plaque with a "sanding" action of the calcified component. Theoretically, OA might have a selective action on the rigid calcified part of the coronary, while the healthy compliant tissue might flex away and be spared. The size of the debris generated during OA is smaller (2 um), thereby making the chance of developing slow flow very minimal. When compared with rotablation, better plaque/lesion modification is associated with OA by producing deeper and longer cuts. True "ablation" of calcium component on OCT has been reported more frequently with $\mathrm{OA}$ than with rotablation.

OA was FDA approved in 2013. Diamondback 360 coronary orbital atherectomy system (Cardiovascular systems, Saint Paul, MN, USA) has single burr of $1.25 \mathrm{~mm}$ with two speeds of 80,000 and 120000 RPM and Glide assist at 5000 RPM, mounted on Viper wire 0.012" with 0.014 " tip, with classic and mini crowns. It is used with Viperslide lubricant and is 6F compatible. High-speed option is be avoided in tortuous lesions and vessel diameter less than $3 \mathrm{~mm}$. A minimum of $10 \mathrm{~mm}$ distance should be maintained between wire tip and crown. Minicrown has diamond-coated tip: useful in ostial or near occlusive lesions. Complications are akin to that of RA, although burr entrapment is rare. Angiographic complications occur in less than $1 \%$. Minicrown was evaluated
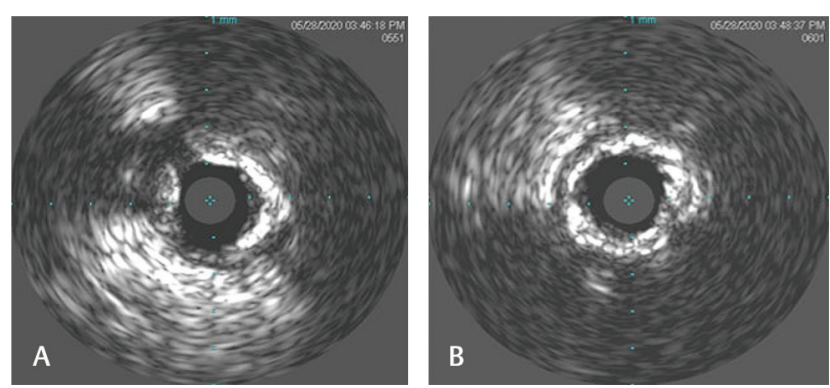

Fig. 1 Post (A) and Pre (B) AngioSculpt $2.5 \mathrm{~mm}$ dilatation in pro left anterior descending (LAD) artery with Agatston score of 208 in LAD (total of 1000) in a multicenter coronary orbital atherectomy system trial (COAST) trial of 100 pts. ORBIT I FIM feasibility trial of 50 pts: prospective, nonrandomized in two sites in India (-Table 2). Q3 Device success 98\% and procedure success 94\%. Comparison with Rotablation: small retrospective or observational studies. Safety and efficacy was established in ORBIT II trial. One study is ongoing: comparison of orbital versus rotational atherectomy effects on coronary microcirculation(ORACLE), which will determine their effect on coronary microcirculation.

Eclipse multicenter trial of 2000 pts compares OA with balloon angioplasty. Results are expected in 2022.33

\section{Intravascular Lithotripsy (IVL)}

IVL is the latest addition to the toolkit against calcific coronary lesions which received a CE mark certification in May 2017 and was established on the principles of renal stone therapy. Sonic pressure waves preferentially impact hard tissues, disrupt calcium, and leave soft tissues undisturbed. Miniaturized and arrayed lithotripsy emitters create a localized field effect by converting electrical energy into transient acoustic pressure pulses at the site of calcium.

The IVL system (Shockwave Medical) is made up of three parts (-Fig. 2): 1) IVL generator: portable and rechargeable, no external connections, quick and easy setup, 2) IVL connector cable: simple magnetic connections, push button activation, and 3) IVL catheter: mounted on workhorse 0.014" guide wire, monorail (RX). The mechanism of action of IVL, as described by Ali et al using OCT, was shown to be an enlargement of the lumen and single or multiple calcium fractures.

The IVL procedure is akin to standard angioplasty requiring minimal or nil learning curve. The shockwave balloon must be sized according to the reference vessel diameter (ratio 1:1). The steps are as follows:

1. IVL catheter crosses the calcific lesion. Integrated balloon positioned across area of interest and expanded to $4 \mathrm{~atm}$ to ensure apposition.

2. Electrical discharge from the emitters vaporizes fluid within the balloon, creating a rapidly expanding and collapsing bubble which generates sonic waves.

3. Waves create an unfocused, circumferential, localized field effect which selectively cracks intimal and medial calcium

4. Balloon can further be inflated to 6 atm (nominal pressure) to optimize lumen gain

Balloon: 12 mm IVL 4 atm, nominal 6 atm, rated 10 atm. Two emitters. one pulse per sec. 80 pulses per catheter

Shockwave C2: Balloon diameters: 2.5, 2.75,3.0,3.25, 3.5, $3.75,4.0 \mathrm{~mm}$. 6F guide compatible, crossing profile 0.043 to 0.046 inches. Energy: 3kV. Pressure: $50 \mathrm{~atm}$. Depth of penetration: $3 \mathrm{~mm} .^{34,35}$

As the shockwave catheter has a higher profile than conventional balloons, predilatation with standard balloons might be necessary in 40 to $45 \%$ of lesions. Plaque modification devices like cutting/scoring balloons/RA/OA can be used concomitantly as adjuvants in challenging cases. Currently, the use of IVL is limited to unstable and stable angina. IVL 
Table 2 Studies of orbital atherectomy

\begin{tabular}{|l|l|l|l|}
\hline & ORBIT I & ORBIT II & REAL WORLD REGISTRY \\
\hline No of pts & 50 & 443 & 458 \\
\hline In-hospital MACE & 4 & 9.8 & - \\
\hline 1 -year MACE & - & 9.7 & 1.8 \\
\hline 1 -year TVR & - & 5.9 & 7.5 \\
\hline
\end{tabular}

Abbreviations: MACE, major adverse cardiac events; TVR, target lesion revascularization.
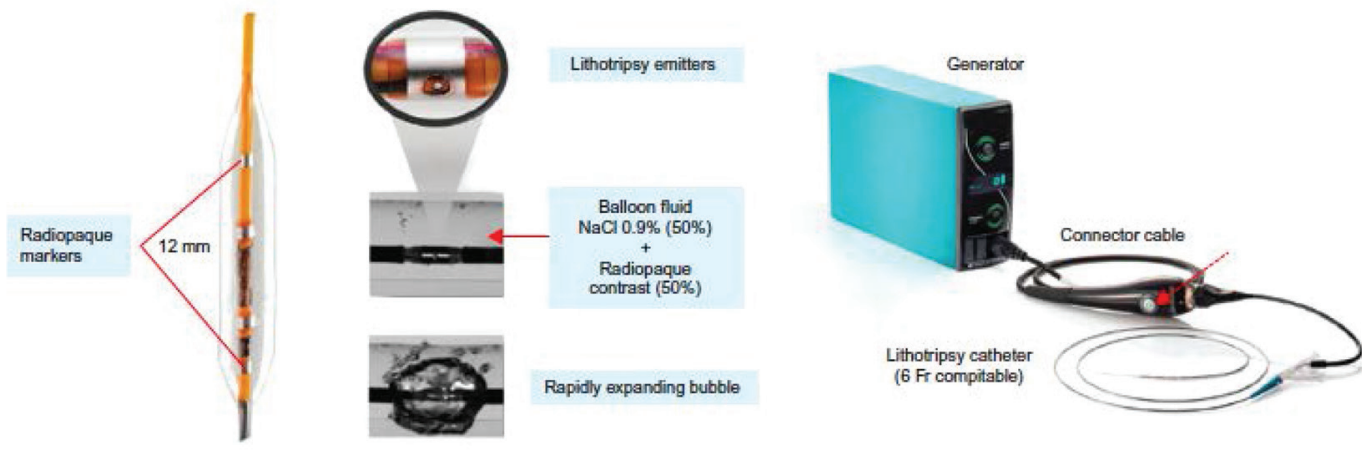

Fig. 2 Intravascular lithotripsy (IVL) system. IVL Generator, connector cable and IVL balloon catheter with 2 emitters

has also been during primary percutaneous transluminal coronary angioplasty (PTCA). ${ }^{36}$ In patients with underexpanded stents due to heavy calcification, the circumferential sonic waves generated by the IVL system have the potential to extend beyond the stent struts and fracture deeper calcium deposits, which may aid in optimal stent expansion and opposition while lowering the complications that may be associated with other methods ( - Fig. $\mathbf{3}$ ).

Electric signals mimicking pacing spikes on the electrocardiogram (ECG) tracing during pulse-delivery have been described. These so-called "shocktopics" and asynchronous cardiac pacing have been reported in a significant number of patients in up to $77.8 \%$ of the cases, with a 16 -fold increased frequency at heart rate $<65 \mathrm{BPM}$. This VOO pacing may be proarrhythmogenic (risk of $\mathrm{R}$ on $\mathrm{T}$ phenomenon), and in patients with pacemakers, an eye needs to be kept on pacemaker function, which should be assessed postprocedure, and inappropriate sensing during the IVL cycles. ${ }^{37,38}$ IVL can trigger supraventricular arrhythmias or paroxysmal atrial fibrillation in susceptible patients undergoing right coronary artery intervention. ${ }^{39}$ There are anecdotal reports of coronary perforation with the use of IVL. ${ }^{40,41}$

IVL is to be used with caution in critical lesions and in very tortuous vessels. In vessel size $>4 \mathrm{~mm}$ or eccentric calcium plaques which prevent appropriate IVL balloon apposition to vessel wall, the success of IVL decreases and other options need to be explored. There are anecdotal reports of its use in unprotected left main disease. .2,43 $^{42}$ Rotatripsy is a hybrid drill and disrupt technique. ${ }^{44-46}$ IVL may also be useful in recalcitrant de novo lesions and in in-stent restenosis. ${ }^{47}$

\section{Clinical Evidence with IVL System}

Disrupt CAD I and II are published. Disrupt III and IV are ongoing. The findings of DISRUPT CAD are summarized in - Table 3. ${ }^{48,49}$

We need large trials and long-term data with IVL before it is incorporated into routine practice.

\section{Excimer Laser}

Although introduced more than two decades ago, its role is presently very limited and is based on the principle of photoablation of atherosclerotic plaque. Laser produces ablation by way of mainly three main mechanisms: 1) photochemical (breaking molecular bonds), 2) photothermal, and 3) photomechanical. When laser acts upon a liquid medium (saline, contrast dye, blood) leading to release of expanding and exploding bubbles that press over the plaque, photomechanical ablation occurs. However, increasing proportions of calcium in a lesion seem to reduce the efficacy of laser. As a result, laser is not advised as the first-line therapy for lesions with severe calcification. ${ }^{50-52}$ The clinical use of coronary laser is limited to being a "bail-out strategy" in uncrossable/undilatable lesions and underexpanded stents. ${ }^{53}$

The CVX 300 System (Philips) uses xenon chloride to produce a light emitted in the ultraviolet $B$ spectrum $(308 \mathrm{~nm}$ ) with a penetration depth of 30 to $50 \mu \mathrm{m}$. A combination of excimer laser and RA was recently described (the RASER technique). ${ }^{54}$

The various modalities for treatment of calcified lesions are summarized in - Table 4 . 


\section{Conclusion}

Armed with the latest advances in the field, interventionalists are approaching calcific lesions today with greater confidence and renewed enthusiasm. The success rates are rising, and complication rates are falling, signaling the onset of a new and exciting era in the management of these hitherto seemingly impossible lesions. Newer generation drug-eluting stents (DES) and more effective antiplatelet agents have greatly reduced the risk of stent thrombosis and restenosis. In clinical practice, we come across a lot of physician inertia and financial constraints because of which most of the dedicated devices to tackle coronary calcium, especially RA, OA and IVL, are grossly underutilized.

IVL is a new and exciting technology that holds great promise for the future. It has a shorter learning curve and
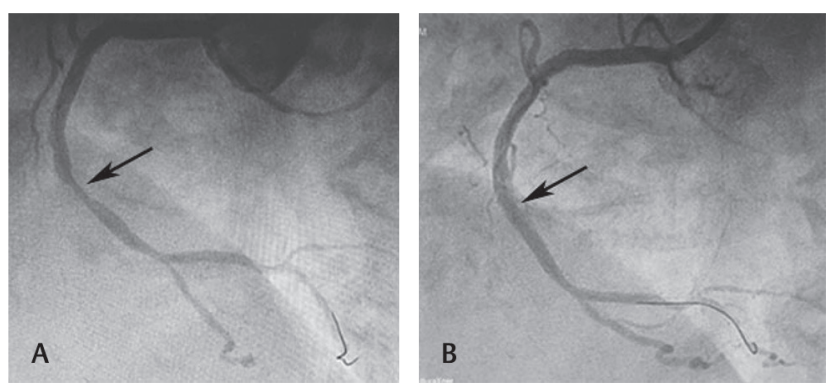

Fig. 3 Use of IVL for underexpanded stent in right coronary artery $($ RCA), pre $(\mathbf{A})$ and post $(\mathbf{B})$ virtually nonexistent complications compared with RA/OA and is currently only being hindered from becoming mainstream due to its cost which is currently proving to be almost prohibitive. Sometimes complex lesions may warrant the use of more than one technique to overcome the calcium (-Fig. 4).

The use of imaging (OCT > IVUS) helps to characterize the calcium and plan the strategy better. It is imperative to remember that a well-planned approach is a job half done. The IVUS/OCT score by Fujino et al helps decide the choice of initial therapy. Lower scores (1-2 points) can go for high or very high pressure balloons, whereas high scores (3-5 points) are better served by IVL. One of the most challenging aspects of planning treatment modalities is that most of the imaging modalities we are dependent on are invasive in nature and hence often throw up surprises while evaluation on table, prompting a change in strategy during procedure. The availability of an accurate noninvasive modality for evaluation of calcific lesions will greatly change the way we approach these lesions in the future.

As healthcare scales newer heights and longevity increases, the problem of calcific coronary stenoses is likely to be encountered by interventionalists with an increasing frequency in the future when dealing with an aging population with multiple comorbidities like diabetes and renal failure. It is essential to be well-versed with the various strategies to tackle these lesions and train ourselves with imaging techniques that will form the basis of lesion assessment and planning. The acronym ROLE (Rotablator, OA, IVL and

Table 3 The DISRUPT CAD studies

\begin{tabular}{|l|l|l|l|}
\hline Disrupt CAD I & $N=60$ & Feasibility study & Success 94\%, MACE 5.8\% \\
\hline Disrupt CAD II & $N=120$ & Safety, efficacy study & $\begin{array}{l}\text { Success 94.2\% 1-month MACE } \\
5.8 \%, \text { non Q MI. }\end{array}$ \\
\hline Disrupt CD II, OCT substudy & 47 & Mechanism of benefit & $\begin{array}{l}\text { Intraplaque ca fracture } 78.7 \%, \\
3.4+/ \square 2.6 \text { per lesion }\end{array}$ \\
\hline Disrupt CAD III & $N=392$ & Ongoing, USA, Europe & For FDA approval \\
\hline Disrupt CAD IV & $N=72$ & Ongoing, Japan & Results 2022 \\
\hline
\end{tabular}

Table 4 Summary of commonly used treatment modalities for tackling calcified coronary lesions

\begin{tabular}{|c|c|c|c|c|}
\hline Modality & Types & Advantages & Disadvantages & Evidence \\
\hline Scoring balloon & $\begin{array}{l}\text { Angiosculpt } \\
\text { NSE } \alpha \\
\text { Scoreflex }\end{array}$ & Easy to use, controlled calcium disruption & $\begin{array}{l}\text { High crossing profile, } \\
\text { not useful in high } \\
\text { calcium burden }\end{array}$ & Meta-analysis \\
\hline Cutting balloon & Flextome & Controlled cutting & High crossing profile & Meta-analysis \\
\hline RA & $\begin{array}{l}\text { Boston } \\
\text { Rota wire }\end{array}$ & Effective, forward, pulverization & $\begin{array}{l}\text { Higher complica- } \\
\text { tions, no side branch } \\
\text { protection }\end{array}$ & Rotaxus \\
\hline OA & $\begin{array}{l}\text { Spectranetics, } \\
\text { Philips, } \\
\text { Viper wire }\end{array}$ & Forward and backward sanding & $\begin{array}{l}\text { Higher complication, } \\
\text { no side branch access }\end{array}$ & Orbit 2 \\
\hline IVL & Shockwave & Controlled Ca rupture in plaque & $\begin{array}{l}\text { Preserved side branch } \\
\text { access }\end{array}$ & $\begin{array}{l}\text { Disrupt CAD I } \\
\text { and II }\end{array}$ \\
\hline Excimer laser & Philips, spectranetics & Unexpanded stents, ISR & & $\begin{array}{l}\text { Element registry } \\
\text { LARS registry }\end{array}$ \\
\hline
\end{tabular}

Abbreviations: ISR, in-stent restenosis; IVL, intravascular lithotripsy; OA, orbital atherectomy; RA, rotational atherectomy. 

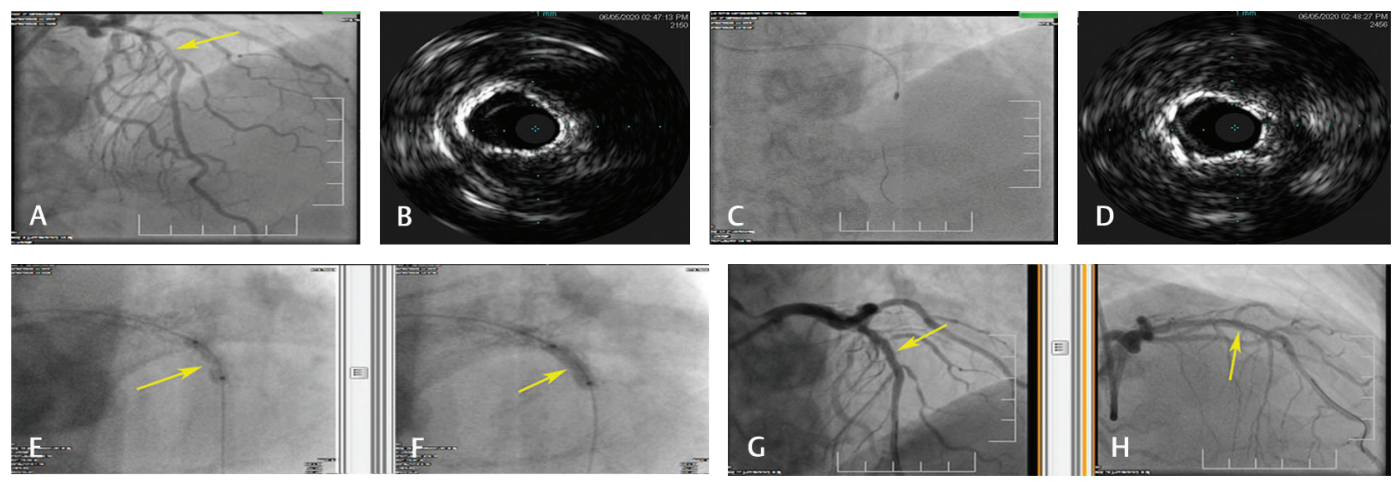

Fig. 4 Patient with a calcific lesion in mid left anterior descending (LAD) artery (yellow arrow, A) as seen on intravascular ultrasound (IVUS) (B), rotablator with $1.25 \mathrm{~mm}$ burr done (C), after rota IVUS (D) showed that the calcium failed to yield. Cutting balloon inflation with Angiosculpt was done $(\mathbf{E})$ after which the calcium yielded $(\mathbf{F})$. Final result $(\mathbf{G}, \mathbf{H})$

Excimer laser) should be recalled in highly calcified coronary lesions. IVL is a transformational therapy, which with its short learning curve and safety even in the hands of relatively inexperienced operators, holds great promise for the future. One can only hope that with increasing use, costs decrease and give this wonderful therapy the spot in the limelight that it truly deserves.

\section{Conflicts of Interest}

None declared.

\section{Acknowledgments}

We thank Dr. PRK Jain for permitting use of images ( - Fig. 3) from his series and Mr. O.B. Chaitanya for helping with figures used in the article

\section{References}

1 Bourantas CV, Zhang YJ, Garg S, et al. Prognostic implications of coronary calcification in patients with obstructive coronary artery disease treated by percutaneous coronary intervention: a patient-level pooled analysis of 7 contemporary stent trials. Heart 2014;100(15):1158-1164

2 Généreux P, Madhavan MV, Mintz GS, et al. Ischemic outcomes after coronary intervention of calcified vessels in acute coronary syndromes. Pooled analysis from the HORIZONS-AMI (Harmonizing Outcomes with Revascularization and Stents in Acute Myocardial Infarction) and ACUITY (Acute Catheterization and Urgent Intervention Triage Strategy). TRIALS. J Am Coll Cardiol 2014;63:1845-1854

3 Nakahara T, Dweck MR, Narula N, Pisapia D, Narula J, Strauss HW. Coronary artery calcification: from mechanism to molecular imaging. JACC Cardiovasc Imaging 2017;10(5): 582-593

4 Mori H, Torii S, Kutyna M, Sakamoto A, Finn AV, Virmani R. Coronary artery calcification and its progression: what does it really mean? JACC Cardiovasc Imaging 2018;11(1):127-142

5 Sage AP, Tintut Y, Demer LL. Regulatory mechanisms in vascular calcification. Nat Rev Cardiol 2010;7(9):528-536

6 Nakahara T, Narula J, Strauss HW. Calcification and inflammation in atherosclerosis: which is the chicken, and which is the egg? J Am Coll Cardiol 2016;67(1):79-80

7 Nakahara T, Strauss HW. From inflammation to calcification in atherosclerosis. Eur J Nucl Med Mol Imaging 2017;44(5): 858-860

8 Faggiano P, Dasseni N, Gaibazzi N, Rossi A, Henein M, Pressman G. Cardiac calcification as a marker of subclinical atherosclerosis and predictor of cardiovascular events: A review of the evidence. Eur J Prev Cardiol 2019;26(11):1191-1204

9 Blaha MJ, Mortensen MB, Kianoush S, Tota-Maharaj R, CainzosAchirica M. Coronary artery calcium scoring: Is it time for a change in methodology? JACC Cardiovasc Imaging 2017; 10(8):923-937

10 Criqui MH, Denenberg JO, Ix JH, et al. Calcium density of coronary artery plaque and risk of incident cardiovascular events. JAMA 2014;311(3):271-278

11 van Rosendael AR, Narula J, Lin FY, et al. Association of high-density calcified $1 \mathrm{~K}$ plaque with risk of acute coronary syndrome. JAMA Cardiol 2020;5(3):282-290

12 Wang X, Matsumura M, Mintz GS, et al. In vivo calcium detection by comparing optical coherence tomography, intravascular ultrasound, and angiography. JACC Cardiovasc Imaging 2017;10(8):869-879

13 Fujino A, Mintz GS, Matsumura M, et al. A new optical coherence tomography-based calcium scoring system to predict stent underexpansion. EuroIntervention 2018;13(18):e2182-e2189

14 Puri R, Nicholls SJ, Shao M, et al. Impact of statins on serial coronary calcification during atheroma progression and regression. J Am Coll Cardiol 2015;65(13):1273-1282

15 Secco GG, Buettner A, Parisi R, et al. Clinical experience with very high-pressure dilatation for resistant coronary lesions. Cardiovasc Revasc Med 2019;20(12):1083-1087

16 van Gaal WJ, Banning AP. Percutaneous coronary intervention and the no-reflow phenomenon. Expert Rev Cardiovasc Ther 2007;5(4):715-731

17 Sakakura K, Funayama H, Taniguchi Y, et al. The incidence of slow flow after rotational atherectomy of calcified coronary arteries: a randomized study of low speed versus high speed. Catheter Cardiovasc Interv 2017;89(5):832-840

18 Cavusoglu E, Kini AS, Marmur JD, Sharma SK. Current status of rotational atherectomy. Catheter Cardiovasc Interv 2004;62(4):485-498

19 Yamamoto $\mathrm{MH}$, Maehara A, Karimi Galougahi K, et al. Mechanisms of orbital versus rotational atherectomy plaque modification in severely calcified lesions assessed by optical coherence tomography. JACC Cardiovasc Interv 2017;10(24): 2584-2586

20 Abdel-Wahab M, Richardt G, Joachem Buttner H, et al. High speed rotational atherectomy before paclitaxal eluting stent implantation in complex calcified coronary lesions: the randomized ROTAXUS trial. J Am Coll Cardiol Intv. 2013;6:10-19

21 Mehanna E, Abbott JD, Bezerra HG. Optimizing percutaneous coronary intervention in calcified lesions: insights from optical coherence tomography of atherectomy. Circ Cardiovasc Interv 2018;11(5):e006813 
22 Barath P, Fishbein MC, Vari S, Forrester JS. Cutting balloon: a novel approach to percutaneous angioplasty. Am J Cardiol 1991;68(11):1249-1252

23 Otsuka Y, Koyama T, Imoto Y, et al. Prolonged inflation technique using a scoring balloon for severe calcified lesion. Int Heart J 2017;58(6):982-987

24 Mauri L, Bonan R, Weiner BH, et al. Cutting balloon angioplasty for the prevention of restenosis: results of the Cutting Balloon Global Randomized Trial. Am J Cardiol 2002;90(10):1079-1083

25 Karvouni E, Stankovic G, Albiero R, et al. Cutting balloon angioplasty for treatment of calcified coronary lesions. Catheter Cardiovasc Interv 2001;54(4):473-481

26 Okura H, Hayase M, Shimodozono S, et al; REDUCE Investigators. Restenosis Reduction by Cutting Balloon Evaluation. Mechanisms of acute lumen gain following cutting balloon angioplasty in calcified and noncalcified lesions: an intravascular ultrasound study. Catheter Cardiovasc Interv 2002;57(4):429-436

27 Tian W, Mahmoudi M, Lhermusier T, et al. Comparison of rotational atherectomy, plain old balloon angioplasty, and cutting-balloon angioplasty prior to drug-eluting stent implantation for the treatment of heavily calcified coronary lesions. J Invasive Cardiol 2015;27(9):387-391

28 Redfors B, Maehara A, Witzenbichler B, et al. Outcomes after successful percutaneous coronary intervention of calcified lesions using rotational atherectomy, cutting-balloon angioplasty, or balloon-only angioplasty before drug-eluting stent implantation. J Invasive Cardiol 2017;29(11):378-386

29 Fonseca A, Costa JdeR Jr, Abizaid A, et al. Intravascular ultrasound assessment of the novel AngioSculpt scoring balloon catheter for the treatment of complex coronary lesions. J Invasive Cardiol 2008;20(1):21-27

30 Ashida K, Hayase T, Shinmura T. Efficacy of lacrosse NSE using the "leopard-crawl" technique on severely calcified lesions. J Invasive Cardiol 2013;25(10):555-564

31 Kawase Y, Saito N, Watanabe S, et al. Utility of a scoring balloon for a severely calcified lesion: bench test and finite element analysis. Cardiovasc Interv Ther 2014;29(2):134-139

32 De Maria GL, Scarsini R, Banning AP. Management of calcific coronary artery lesions: is it time to change our interventional therapeutic approach? JACC Cardiovasc Interv 2019;12(15):1465-1478

33 Shlofmitz E, Jeremias A, Shlofmitz R, Ali ZA. Lesion preparation with orbital atherectomy. Interv Cardiol (Lond) 2019;14(3):169-173

34 Sorini Dini C, Nardi G, Ristalli F, Mattesini A, Hamiti B, Di Mario C. Contemporary approach to heavily calcified coronary lesions. Interv Cardiol (Lond) 2019;14(3):154-163

35 Forero MNT, Daemen J. The coronary intravascular lithotripsy system. Interv Cardiol (Lond) 2019;14(3):174-181

36 Wong B, El-Jack S, Newcombe R, et al. Shockwave intravascular lithotripsy of calcified coronary lesions in ST elevation myocardial infarction: first in man experience. J Invasive Cardiol 2019;31(5):E73-E75

37 McQuillan C, Alkhalil M, Johnston PW. A paced heart without a pacemaker. Eur Heart J 2019;40(10):819a

38 Wilson SJ, Spratt JC, Hill J, et al. Incidence of "Shocktopics" and asynchronous cardiac pacing in patients undergoing coronary intravascular lithotripsy. EuroIntervention 2020 (e-pub ahead of print). doi:10.4244/EIJ-D-19-00484
39 Curtis E, Khan A, El-Jack S, Glenie T. Precipitation of de novo atrial fibrillation during Shockwave Intravascular Lithotripsy after pacing capture during the treatment of proximal right coronary artery disease: a case report. Eur Heart J Case Rep 2019;3(4):1-4

40 López-Lluva MT, Jurado-Román A, Sánchez-Pérez I, Abellán-| Huerta J, Lozano Ruíz-Poveda F. Shockwave useful but potentially dangerous. JACC Cardiovasc Interv 2019;12(5): 500-501

41 Simsek C, Vos J, IJsselmuiden A, et al. Coronary artery perforation after shockwave intravascular lithotripsy. J Am Coll Cardiol 2020;2(2):247-249

42 Wong B, EL-Jack S, Khan A, et al. Treatment of calcified unprotected left main disease with lithotripsy: the first case series. J Invasive Cardiol 2019;31(5):E73-E75

43 Salazar CH, Travieso A, Gonzalo N, Escaned J. Intracoronary lithotripsy in percutaneous treatment of calcific left main coronary stenoses. J Am Coll Cardiol 2019;1(1):46-49

44 Aznaouridis K, Bonou M, Masoura C, Kapelios C, Tousoulis D, Barbetseas J. Rotatripsy: a hybrid drill and disrupt approach for treating heavily calcified coronary lesions. J Invasive Cardiol 2020;32(6):E175

45 Jurado-Román A, Gonzálvez A, Galeote G, Jiménez-Valero S, Moreno R. RotaTripsy: combination of rotational atherectomy and intravascularlithotripsy for the treatment of severely calcified lesions. JACC Cardiovasc Interv 2019;12(15): e127-e129

46 Chen G, Zrenner B, Pyxaras SA. Combined rotational atherectomy and intravascular lithotripsy for the treatment of severely calcified in-stent neoatherosclerosis: a mini review. Cardiovasc Revasc Med 2019;20(9):819-821

47 Alfonso F, Bastante T, Antuña P, et al. Coronary lithotripsy for the treatment of undilatable calcified de nono and instent restenosis lesions. JACC Cardiovasc Interv 2019;12(5):497-499

48 Brinton TJ, Ali ZA, Hill JM, et al. Feasibility of shockwave coronary intravascular lithotripsy for the treatment of calcified coronary stenoses. Circulation 2019;139(6):834-836

49 Ali ZA, Nef H, Escaned J, et al. Safety and effectiveness of coronary intravascular lithotripsy for treatment of severely calcified coronary stenoses: the Disrupt CAD II Study. Circ Cardiovasc Interv 2019;12(10):e008434

50 Goldberg SL, Colombo A, Akiyama T. Stent under-expansion refractory to balloon dilatation: a novel solution with excimer laser. J Invasive Cardiol 1998;10(5):269-273

51 Bilodeau L, Fretz EB, Taeymans Y, Koolen J, Taylor K, Hilton DJ. Novel use of a high-energy excimer laser catheter for calcified and complex coronary artery lesions. Catheter Cardiovasc Interv 2004;62(2):155-161

52 Bittl JA. Clinical results with excimer laser coronary angioplasty. Semin Interv Cardiol 1996;1(2):129-134

53 Lee T, Shlofmitz RA, Song L, et al. The effectiveness of excimer laser angioplasty to treat coronary in-stent restenosis with peri-stent calcium as assessed by optical coherence tomography. EuroIntervention 2019;15(3):e279-e288

54 Fernandez JP, Hobson AR, McKenzie D, et al. Beyond the balloon: excimer coronary laser atherectomy used alone or in combination with rotational atherectomy in the treatment of chronic total occlusions, non-crossable and non-expansible coronary lesions. EuroIntervention 2013;9(2):243-250 